\title{
Analysis of Two-Phase Flow inside Porous Diffuser
}

\author{
Omar Rafae Alomar ${ }^{1}$, Ibrahim Atiya Mohamed $^{1}$, Qais Abid Yousif ${ }^{1}$ \\ (omar.alomar@ntu.edu.iq, ibams_1962@ntu.edu.iq, kaisyusuf@ntu.edu.iq) \\ ${ }^{1}$ Northern Technical University, Engineering Technical College of Mosul, Cultural Group Street, \\ Mosul, Iraq
}

\begin{abstract}
Evaporation process inside diffuser filled with porous medium is numerically investigated. The governing equations have been made dimensionless form and discretised using Finite Volume Method (FVM). Effects of the relevant parameters on the temperature distribution and liquid saturation have been carefully analysed. Numerical results showed that the smoothing treatment of effective diffusion coefficient promote the undesired "jump" in the temperature profile. Furthermore, the inlet Reynolds number, heat flux, outlet radius, length of spread section, and pipe length have a strong effect on the beginning and ending of evaporation process, while porosity and Darcy number have slight influence. Finally, the investigation exhibits a helpful instrument for structuring a diffuser evaporator with goal to hold the system under safety conditions.
\end{abstract}

Keywords: porous diffuser, two-phase flow, phase change process

\section{Introduction}

Two-phase flow with water phase change inside absorbent media are attractive subject for engineers in many important technological applications [1]. Two-phase flow within porous media was traditionally solved using Two-Phase Mixture Model (TPMM) [1, 2]. For ease of numerical implementation, TPMM [3] has been utilized in simulations of two-phase flow within porous media [3 - 8]. TPMM has been developed according to Local Thermal Equilibrium (LTE) condition.

However, the use of TPMM [2] faced serious problems due to the appearance of a sharp "jump" in the expected temperature profile within a very short distance, mainly due to the discontinuity in the effective diffusion coefficient (EDC). To overcome this problem, Alomar et al. [8] proposed a successful remedy (smoothing algorithm) for EDC and they presented several case studies. Later, they employed this remedy for prediction of two-phase flow in a circular porous evaporator [9 - 11] and porous channel [12] using both LTE and Non-Equilibrium (LTNE) conditions. Ray and Alomar [13] developed a modified formulation to eliminate the drawbacks in the original enthalpy (h) formulation of TPMM [1]. They found that modified h-formulation in comparison with volumetric enthalpy $(\mathrm{H})$ formulation [2] requires significantly less computational time. Therefore, they recommended it for the simulation of two-phase flow within porous media. After that, the modified h-formulation along with LTNE model has been used to demonstrate the effectiveness of non-staggered grid layouts [14] and to analyse of anisotropy to define the porous media properties [15]. Recently, 
the modified h-formulation has been also used by Alomar et al. [16] in order to inspect the effect of inclination angle of the channel while simulating incomplete phase change process.

The interesting feature of complete evaporation process inside porous media is the density of water reduces considerably from the liquid phase to the vapour phase by approximately 1600 times and hence, the vapour phase takes notably wider volume. To conserve the continuity of mass during the phase change process, the velocity sharply increases through channel of constant area in the axial direction. Thus, it may be worthwhile to use a diffuser duct. In this regards, the main purpose of this work is to numerically investigate the two-phase flow with liquid phase change inside diffuser duct to inspect the influence of variable crosssectional area of the duct. The mathematical model is applied using h-formulation [13] in onedimensional problems. It can be mentioned here that the one-dimensional model is quite often used as an idealisation while simulating two-phase flows inside porous media. Effects of relevant parameters on the temperature and liquid saturation profiles have been carefully considered.

\section{Mathematical Forumlation}

\subsection{Problem Description}

The schematic representation of the geometry is given in Fig. 1. The problem under investigation is the complete evaporation process inside circular porous diffuser duct. The flow has been considered to be one-dimensional under steady state condition. An asymmetric heat source $\dot{q}_{w}^{\prime \prime}$ has been applied on the diffuser wall. An external pressure difference drives the water at the inlet to flow inside the diffuser with uniform velocity $u_{\text {in }}$ and low temperature $T_{i n}\left(T_{i n}<T_{\text {sat }}\right)$. The water is directly heated upon entry the diffuser. With adequately increasing the heat flux, boiling happens close to the heated surface and hence, a superheated vapour region is formed.

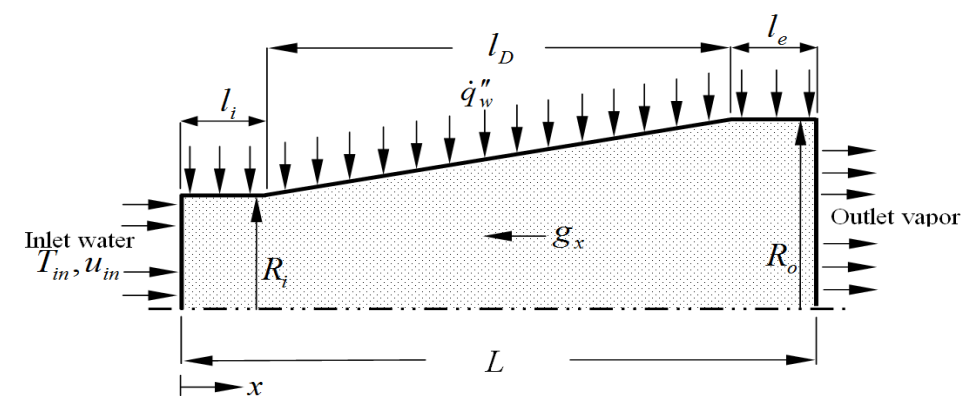

Fig. 1 Geometry of a porous diffuser duct

\subsection{Governing Conservation model}

In this work, the assumption of LTE is used, which assumes the fluid and solid phases coexist at the same temperature. Since the present problem has been considered under low 
velocity, the modified h-formulation has been presented based on Darcy law. The conservation equations are listed in Table 1 based on dimensionless representation. All dimensions have been scaled with the inlet radius $\left(R_{i}\right)$ as given in Fig. 1, whereas all the velocities have been scaled with the inlet velocity $\left(u_{i n}\right)$. By integrating the two-dimensional governing equations over the entire cross-sectional area of the diffuser, the steady, onedimensional form conservation equations of mass, momentum, and energy are obtained as:

$$
\begin{gathered}
\frac{d}{d x^{*}}\left(\rho^{*} u^{*} A_{c}^{*}\right)=0 \\
\rho^{*} u_{x}^{*}=-\frac{K^{*}}{v^{*}}\left(\frac{d p^{*}}{d x^{*}}-\rho_{k}^{*} b_{x}^{*}\right) \\
\frac{d}{d x^{*}}\left(\gamma_{h} \rho_{x}^{*} u_{x}^{*} h^{*}\right)=\frac{d}{d x^{*}}\left(\Gamma_{h}^{*} \frac{d h^{*}}{d x^{*}}\right)+\frac{d}{d x^{*}}\left[f \frac{K^{*} \Delta \rho^{*} h_{f g}^{*}}{v_{v}^{*}} b_{x}^{*}\right]+\frac{P^{*}}{A_{c}^{*}} \dot{Q}_{w}^{*}
\end{gathered}
$$

Table 1. Variables in non-dimensional form $[9,13,17]$

\begin{tabular}{ll}
\hline Dimensionless variables & Expressions \\
\hline Length & $x^{*}=x / R_{i} ; r^{*}=r / R_{i}$ \\
Density & $\rho^{*}=\rho / \rho_{l} ; \rho_{l}^{*}=1$ \\
Velocity & $u^{*}=u / u_{i n}$ \\
Temperature & $T^{*}=T C_{p l} / h_{f g}$ \\
Enthalpy & $h^{*}=h / h_{f g} ; h_{f g}^{*}=1$ \\
Pressure & $p^{*}=p / \rho_{l} u_{i n}^{2}$ \\
Dynamic viscosity & $\mu^{*}=\mu / \mu_{l} R e_{l} ; \mu_{l}^{*}=1 / \operatorname{Re}_{l}$ \\
Thermal conductivity & $k^{*}=k / \rho_{l} C_{p l} R_{i} u_{i n}=k / k_{l} P e_{l}$ \\
Body force per unit mass & $b_{x}^{*}=\tilde{b}_{x} g R_{i} / u_{i n}^{2}=\tilde{b}_{x} / F r^{2}$ \\
Heat flux & $\dot{q}_{w}^{\prime \prime *}=\dot{q}_{w}^{\prime \prime} / \rho_{l} u_{i n} h_{f g}=Q_{w}^{*} / R e_{l}$ \\
Isobaric Expansion & $\beta^{*}=\beta h_{f g} / C_{p l}$ \\
Permeability or Darcy number & $K^{*}=K / R_{i}^{2}$ \\
\hline
\end{tabular}

In Eqs. (1) and (3), $A_{c}^{*}=\left(R^{*}\right)^{2} / 2$ is the cross-sectional area and $P^{*}=R^{*}$ represents the perimeter. Furthermore, $b_{x}^{*}$ represents the dimensionless body force per unit mass and it is considered equal to -1 . All the definitions that showing in Eqs. 1 to 3 are supplied in Tables 1 and 2. The Darcy number $K^{*}=K / R_{i}^{2}$ ( $K$ is permeability in dimensional form) has been determined as a function of porosity $\varepsilon$ and average pore diameter $d_{p}$ [18]. $\rho_{k}^{*}$ is the kinetic density given by [17]:

$$
\rho_{k}^{*}=\rho_{l}^{*}\left[1-\beta_{l}^{*}\left(T^{*}-T_{s a t}^{*}\right)\right] \lambda_{l}+\rho_{v}^{*}\left[1-\beta_{v}^{*}\left(T^{*}-T_{s a t}^{*}\right)\right] \lambda_{v}
$$


Table 2. Mixture properties in two-phase zone [13]

\begin{tabular}{ll}
\hline Variables & Expressions \\
\hline Density & $\rho^{*}=\rho_{l}^{*} s+\rho_{v}^{*}(1-s)$ \\
Enthalpy & $\rho^{*} h^{*}=h_{l}^{*} \rho_{l}^{*} s+(1-s) \rho_{v}^{*} h_{v}^{*}$ \\
Kinematic Viscosity & $\left.v^{*}=\left[k_{r l} / v_{l}^{*}+k_{r v} / v_{v}^{*}\right]^{-1}\right]\left(h_{v, s a t}^{*}-\lambda_{l} h_{f g}^{*}\right)$ \\
Advection Correction Coefficient & $\gamma_{h}=\frac{\left[\rho_{l}^{*} s+\rho_{v}^{*}(1-s)\right.}{\rho_{l}^{*} h_{l, s a t}^{*} s+\rho_{v}^{*} h_{v, s a t}^{*}(1-s)}$ \\
Effective Diffusion Coefficient & $\Gamma_{h}^{*}=\frac{\rho^{*} h_{f g}^{*}}{\rho_{v}^{*}\left(h_{v, s a t}^{*}-h^{*}\right)+\rho_{l}^{*}\left(h^{*}-h_{l, s a t}^{*}\right)} D^{*}+k_{e f f}^{*} \frac{d T^{*}}{d h^{*}}$ \\
Capillary Diffusion Coefficient & $D^{*}=\frac{\left(\varepsilon K^{*}\right)^{1 / 2} \sigma^{*}}{\mu_{l}^{*}} \frac{k_{r r} k_{r l}}{\left(v_{v}^{*} / v_{l}^{*}\right) k_{r l}+k_{r v}}\left[-J^{\prime}\right]$ \\
Relative Mobilities & $\lambda_{l}(s)=\frac{k_{r l} / v_{l}^{*}}{k_{r l} / v_{l}^{*}+k_{r v} / v_{v}^{*}} ; \lambda_{v}(s)=\frac{k_{r v} / v_{v}^{*}}{k_{r l} / v_{l}^{*}+k_{r v} / v_{v}^{*}}$ \\
Hindrance Function & $f=\frac{k_{r l} k_{r v} / v_{v}^{*}}{k_{r l} / v_{l}^{*}+k_{r v} / v_{v}^{*}}$ \\
Relative Permeabilities & $k_{r l}=s^{n}, k_{r v}=(1-s)^{n} ; n=1$ \\
Capillary Pressure & $J=1.417(1-s)-2.120(1-s)^{2}+1.263(1-s)^{3}$ \\
\hline
\end{tabular}

Where the effective thermal conductivity is $k_{e f f}^{*}$ that is given in Equ 3. A simple estimation for $k_{\text {eff }}^{*}$ can be written as follows [13]:

$$
k_{e f f}^{*}=(1-\varepsilon) k_{s}^{*}+\varepsilon\left[k_{l}^{*} s+(1-s) k_{v}^{*}\right]
$$

where $k_{l}^{*}, k_{v}^{*}$ and $k_{s}^{*}$ are the thermal conductivity of liquid, vapour and solid, respectively. The $T^{*}$ in the liquid and superheated vapour regions and the liquid saturation $S$ in two-phase zone can be determined from Table 3 .

Table 3. Relationships of temperature and liquid saturation in the single and two-phase regions [17]

\begin{tabular}{llll}
\hline$h^{*}$ & $T^{*}$ & $s$ & $d T^{*} / d h^{*}$ \\
\hline$h^{*} \leq h_{l, s a t}^{*}$ & $T^{*}=T_{s a t}^{*}+\frac{h^{*}-h_{l, s a t}^{*}}{C_{p l}^{*}}$ & 1 & $1 / C_{p l}^{*}$ \\
$h_{l, \text { sat }}^{*}<h^{*} \leq h_{v, \text { sat }}^{*}$ & $T_{\text {sat }}^{*}$ & $\frac{\rho_{v}^{*}\left(h_{v, s a t}^{*}-h^{*}\right)}{\rho_{v}^{*}\left(h_{v, \text { sat }}^{*}-h^{*}\right)+\rho_{l}^{*}\left(h^{*}-h_{l, s a t}^{*}\right)}$ & 0 \\
$h_{v, \text { sat }}^{*}<h^{*}$ & $T^{*}=T_{s a t}^{*}+\frac{h^{*}-h_{v, s a t}^{*}}{C_{p v}^{*}}$ & 0 & $1 / C_{p v}^{*}$ \\
\hline
\end{tabular}

\subsection{Boundary Conditions}

Boundary conditions in dimensionless form that have been applied at the inlet and exit of the diffuser for one-dimensional model to compute Eqs. (1) - (3) are obtained as: 
i) At diffuser inlet, i.e., $x^{*}=0$, water enters with average velocity $u_{\text {in }}^{*}$ at $T_{i n}^{*}<T_{\text {sat }}^{*}$.

Therefore, the boundary condition is given as:

$$
\begin{aligned}
& h_{i n}^{*}=h_{l, s a t}^{*}+C_{p l}^{*}\left(T_{i n}^{*}-T_{\text {sat }}^{*}\right) \\
& u_{\text {in }}^{*}=1 \\
& \rho_{\text {in }}^{*}=1
\end{aligned}
$$

ii) At diffuser outlet, i.e., $x^{*}=L / R_{i}$, the following boundary condition has been used:

$$
\frac{\partial^{2} h^{*}}{\partial x^{* 2}}=0
$$

The outlet enthalpy $h^{*}$ that required to solve Eq. (3) has been obtained by linear extrapolation. Since the present problem is considered as steady-state in which the mass flowrate and velocity at the cell faces have been obtained as:

$$
\begin{aligned}
& \dot{m}_{c f}^{*}=\dot{m}_{i n}^{*}=\rho_{c f}^{*} u_{c f}^{*} A_{c}^{*} \\
& u_{c f}^{*}=1 /\left(\rho_{c f}^{*} A_{c}^{*}\right)
\end{aligned}
$$

where $\rho_{c f}^{*}$ at the cell face is evaluated using the value of $s$ according to Table 2.

\section{Numerical Solution}

In the discretization of the energy conservation (3), finite volume method on a uniform mesh has been utilized [19]. Upwind and Central Differencing Schemes employed for discretisation the main variables in the energy equation, respectively. At the cell faces, the harmonic mean approximation [19] and linear interpolation method have been used to evaluate $\Gamma_{h}^{*}$ and $\gamma_{h}$ as well as $f$, respectively. To ensure converged solutions, an under relaxation factor 0.1 is required to reach convergence owing to the presence of the energy equation that is non-linear from the relation of interdependence of dependent variable. The convergence is enforced with its criterion set to $10^{-5}$. A grid independence study has been performed as observed in Fig. 2, which shows that a mesh of $N_{C V}=2000$ control volumes produce a grid-independent solution of temperature. 


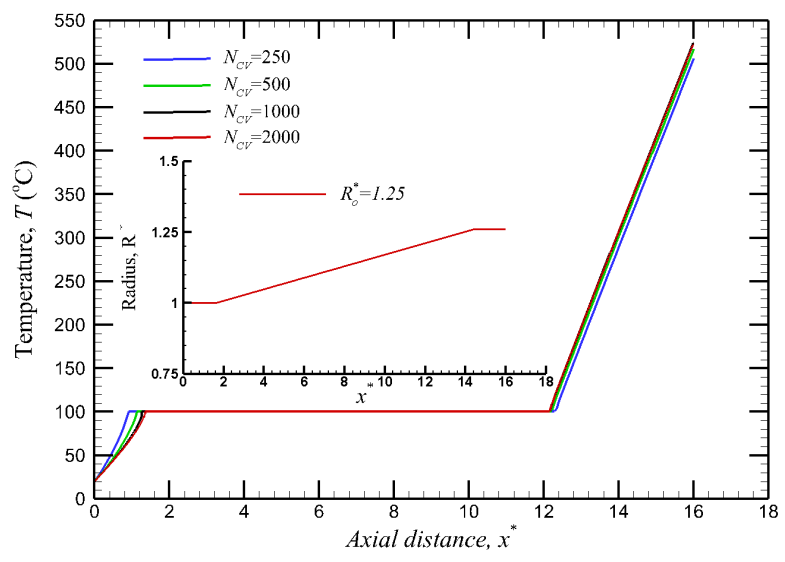

Fig. 2 Axial temperature profiles for various control volumes $N_{C V}$

\section{Results and Discussion}

The inlet radius of the diffuser $R_{i}=25 \mathrm{~mm}$ (i.e., $R_{i}^{*}=1$ ) has kept constant all over the test model, whereas the outlet radius $\left(R_{o}\right)$ varying between $28 \mathrm{~mm}$ and $35 \mathrm{~mm}$. The length of the diffuser $L$ has been considered to vary between $300 \mathrm{~mm}$ and $400 \mathrm{~mm}$. The length of the spread section $\left(l_{D}\right)$, which depends on $l_{i}$, varies between $0.4 \mathrm{~L}$ and $0.8 \mathrm{~L}$, since $l_{e}=0.1 \mathrm{~L}$ has been kept fixed. Sub-cooled liquid water at $T_{i n}=20^{\circ} \mathrm{C}$ has been assumed to enter the diffuser as stated in Table 4, for which $\mathrm{T}_{\text {sat }}$ was considered to be $100^{\circ} \mathrm{C}$ [20].

Table 4. Water charetistics [17]

\begin{tabular}{lll}
\hline Property & Liquid & Vapor \\
\hline Density, $\rho\left(\mathrm{kg} / \mathrm{m}^{3}\right)$ & 957.85 & 0.5978 \\
Specific heat, $C_{p}(\mathrm{~J} / \mathrm{kgK})$ & 4190.2 & 2029.0 \\
Dynamic viscosity, $\mu(\mathrm{kg} / \mathrm{ms})$ & $2.79 \times 10^{-4}$ & $1.202 \times 10^{-5}$ \\
Thermal conductivity, $k(\mathrm{~W} / \mathrm{mK})$ & 0.68 & 0.0248 \\
Expansion coefficient, $\beta\left(\mathrm{K}^{-1}\right)$ & $5.23 \times 10^{-4}$ & $2.4 \times 10^{-3}$ \\
Saturation enthalpy, $h(\mathrm{~J} / \mathrm{kg})$ & $419.02 \times 10^{3}$ & $2676.05 \times 10^{3}$ \\
Latent heat of evaporation, $h_{f g}(\mathrm{~J} / \mathrm{kg})$ & $2257.03 \times 10^{3}$ \\
Surface tension coefficient, $\sigma(\mathrm{N} / \mathrm{m})$ & \multicolumn{2}{c}{0.0589} \\
\hline
\end{tabular}

The average inlet velocity $u_{i n}$ is permitted to change between $1 \times 10^{-4} \mathrm{~m} / \mathrm{s}$ and $5 \times 10^{-4} \mathrm{~m} / \mathrm{s}$. For the porous medium, $k_{s}$ has been taken as $20 \mathrm{~W} / \mathrm{mK}\left(k_{s}^{*}=8.25\right)$ as the one used from low-carbon steel mesh structures, whereas $\varepsilon$ has been assumed to vary between 0.2 and 0.4. The permeability has been assumed to follow the Carman-Kozeny relation [20] and 
hence, the average pore diameter $\left(d_{p}\right)$ has been varied from $0.1 \mathrm{~mm}$ to $0.4 \mathrm{~mm}$. A constant heat flux $\dot{q}_{w}^{\prime \prime}$ has been imposed at the diffuser wall and hence, it has been allowed to varying between $15000 \mathrm{~W} / \mathrm{m}^{2}$ and $20000 \mathrm{~W} / \mathrm{m}^{2}$. Table 5 presents the ranges of dimensionless parameters. The reference parameters are computed by following: $R e_{l}=20, L^{*}=16, \varepsilon=0.3, K^{*}=10^{-8}$, $Q_{w}^{*}=8.5 \times 10^{-1}, l_{D}^{*}=0.8 L^{*}$ and $R_{o}^{*}=1.25$.

Table 5. Parameters in dimensionless form

\begin{tabular}{ll}
\hline Parameters & Ranges \\
\hline Inlet Reynolds number, $R e_{l}$ & $17.5-25$ \\
Heat flux, $Q_{w}^{*}$ & $8 \times 10^{-1}-9 \times 10^{-1}$ \\
Length, $L^{*}$ & $12-16$ \\
Length of spread section, $L_{D}^{*}$ & $0.4 L^{*}-0.8 L^{*}$ \\
Outlet radius, $R_{o}^{*}$ & $1.15-1.4$ \\
Porosity, $\varepsilon$ & $0.2-0.4$ \\
Darcy number, $K^{*}$ & $5 \times 10^{-9}-5 \times 10^{-8}$ \\
\hline
\end{tabular}

\subsection{Varification the Smoothing Treatment}

The treatment process that established by Alomar et al. [8] is used for $\Gamma_{h}^{*}$ to eliminate the sharp jump in the temperature profiles that occurred owing to discontinuity in $\Gamma_{h}^{*}$, particularly at saturated vapour condition. To establish the validity of this treatment process, solutions of two-phase flow calculated with and without remedy of $\Gamma_{h}^{*}$ have been compared as shown in Fig. 3, displays the variations of axial temperature profiles for various $R_{o}$ (i.e., diffuser angle). It can be concluded from Fig. 3 that the treatment process is successfully eliminated the jump in the temperature profiles without alter the solution when compared to the solution without smoothing. Therefore, all subsequent results have been performed using this successful treatment process.

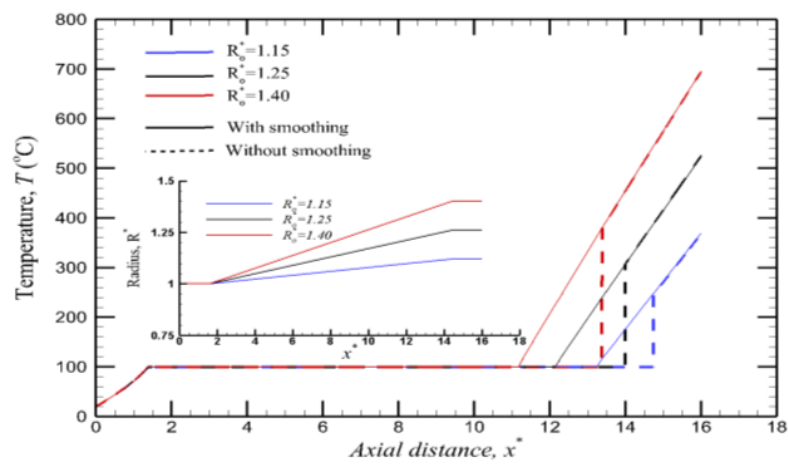

Fig. 3 Temperature profiles with and without remedy of $\Gamma_{h}^{*}$ for various $R_{o}^{*}$ at $R e_{l}=20, \varepsilon=0.3$,

$$
Q_{w}^{*}=8.5 \times 10^{-1} \text { and } K^{*}=10^{-8}
$$




\subsection{Effects of Heating and Flow Condtions}

Figures 4 and 5 illustrate the variations in $T$ and $\mathrm{s}$ for various $Q_{w}^{*}$ (in Fig. 4) and $\operatorname{Re}_{l}$ (in Fig. 5). As $Q_{w}^{*}$ is progressively increased in Fig. 4, mixture region is extended inside the duct and the superheated zone quickly expands towards inside the spread section leading to reduction in the liquid saturation form $s=1$ (sub-cooled liquid region) to $s=0$ (superheated region). The considerable temperature gradient in the sub-cooled region is increased with increasing in $Q_{w}^{*}$ due to increase the diffusion in the upstream section. In addition, $T_{\max }$ at the outlet is significantly increased. Nevertheless, $T_{\max }$ is not exceeded the melting point of the metal that form the structure of the porous medium. Fig. 5 proves that decreasing in $R e_{l}$ leads to higher outlet temperature. This is quite expected and can be explained by increasing the flow resistance. Furthermore, the increase in $R e_{l}$ in Fig. 5(b) leads to delay the water boiling and hence reduces the area of superheated region due to more heat is needed in order to evaporate the water. It can be concluded from Figs. 4 and 5 that the heating and flow conditions required to be properly designed to reach a desired outlet temperature.

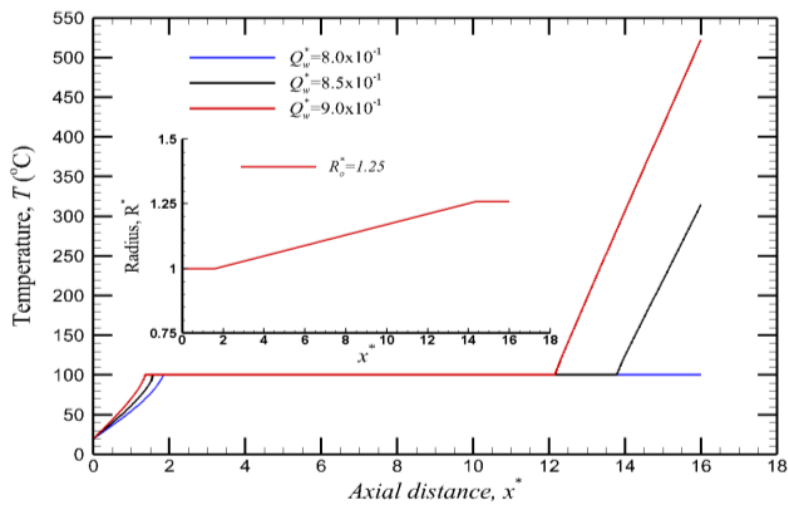

(a)

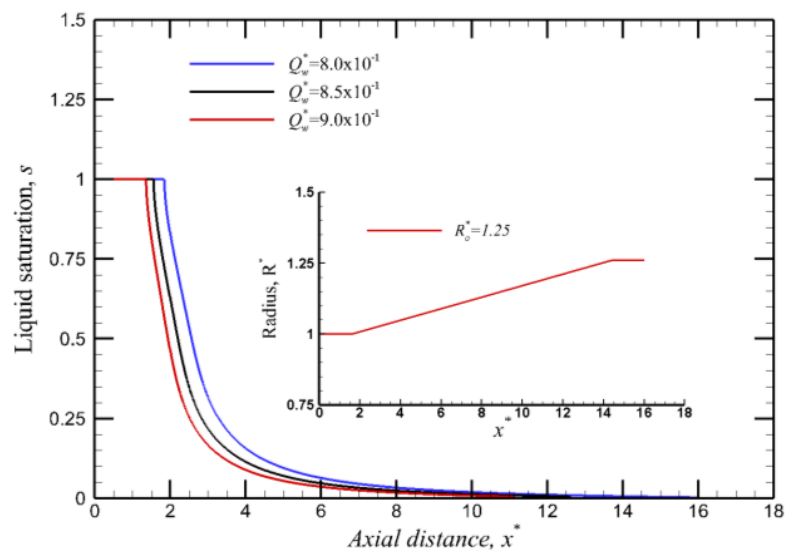

(b)

Fig. 4 Varaitions of axial a) $T$ and b) $s$ for various $Q_{w}^{*}$ at $l_{D}^{*}=0.8 L^{*}, \varepsilon=0.3, R e_{l}=20$, and $K^{*}=10^{-8}$ 


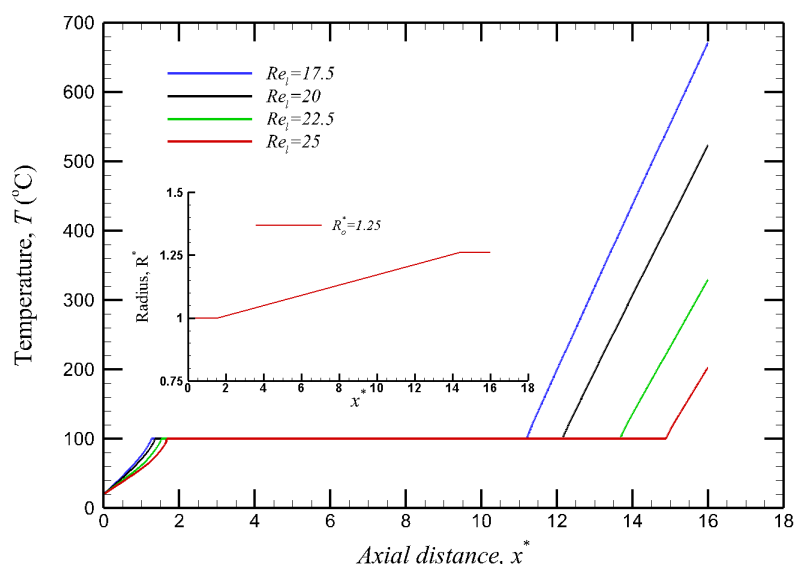

(a)

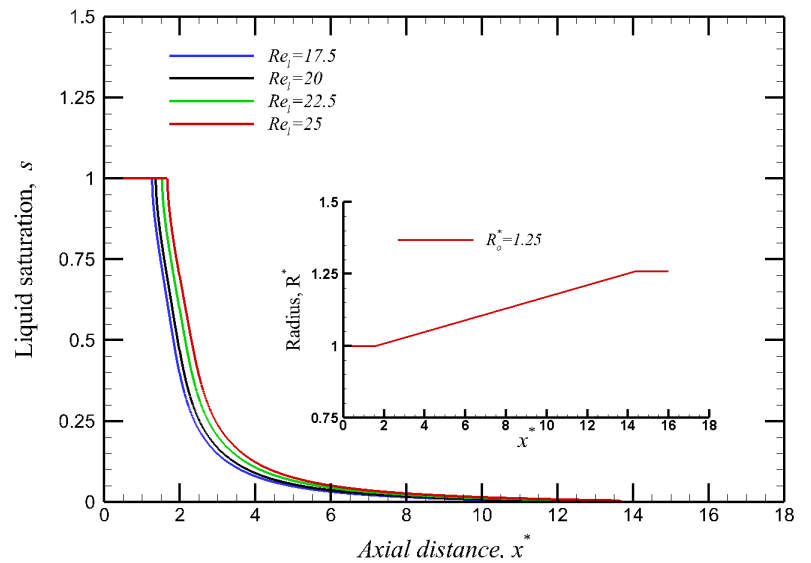

(b)

Fig. 5 Varaitions of axial a) $T$ and b) $s$ for various $R e_{l}$ at $l_{D}^{*}=0.8 L^{*}, \varepsilon=0.3, Q_{w}^{*}=8.5 \times 10^{-1}$, and

$$
K^{*}=10^{-8}
$$

\subsection{Effects ofDarcy Number and Porosity}

Figures 6 and 7 display the variations of $T$ and $s$ within porous evaporator for different $K^{*}$ (in Fig. 6) and $\varepsilon$ (in Fig. 7). Fig. 6 shows that the effect of $K^{*}$ is more pronounced in the liquid zone as compared to the superheated vapour region in which, $\Gamma_{h}^{*}$ is increased only in the mixture zone with increasing in $K^{*}$ and therefore, initiate the process to be changed (see Fig. 6(b)) for higher $K^{*}$. An increased in $\varepsilon$ enhances $\Gamma_{h}^{*}$ in the two-phase zone by increasing $D^{*}$ and decreases $\Gamma_{h}^{*}$ in the liquid and superheated regions by decreasing $k_{e f f}^{*}$. Therefore, the effect of $\varepsilon$ in Fig. 7 is more pronounced on $T$ and $s$ as compared to $K^{*}$. With increasing in $\varepsilon$, the process of phase change terminates later indicating that the mixture zone is expanded in the downstream direction. The later termination of phase change process with increasing in $\varepsilon$ can be explained by decreasing the energy diffused across the saturated vapour condition. The maximum temperature at the outlet of the diffuser remains approximately unaffected within increasing in $K^{*}$. 


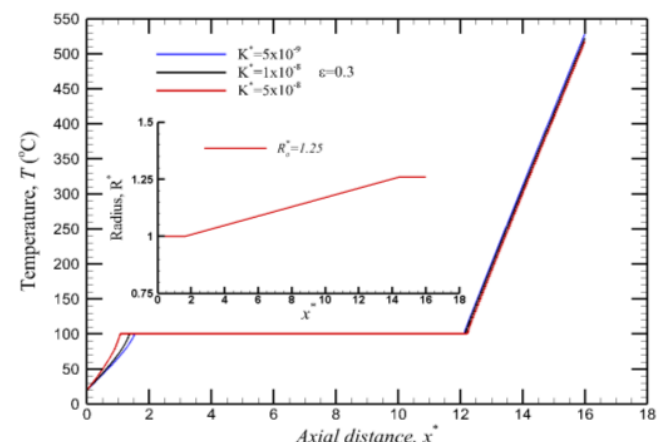

(a)

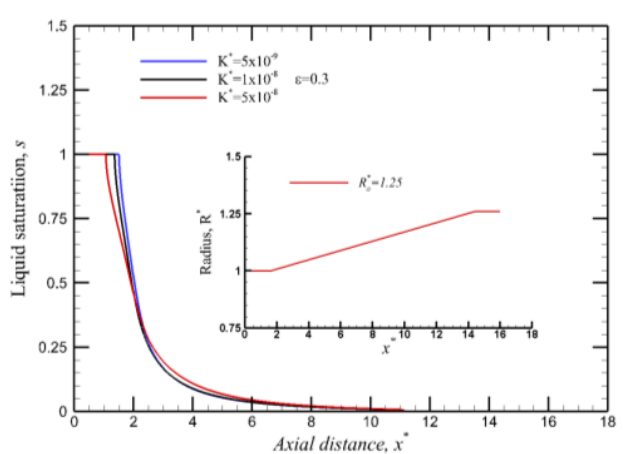

(b)

Fig. 6 Varaitions of axial a) $T$ and b) $s$ for various $K^{*}$ at $l_{D}^{*}=0.8 L^{*}, \varepsilon=0.3, Q_{w}^{*}=8.5 \times 10^{-1}$ and $R e_{l}=20$

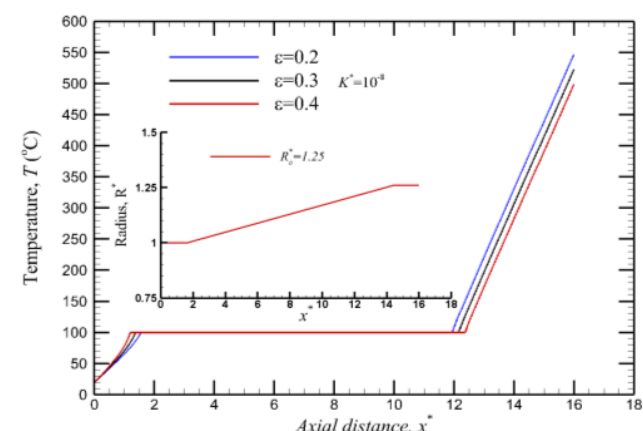

(a)

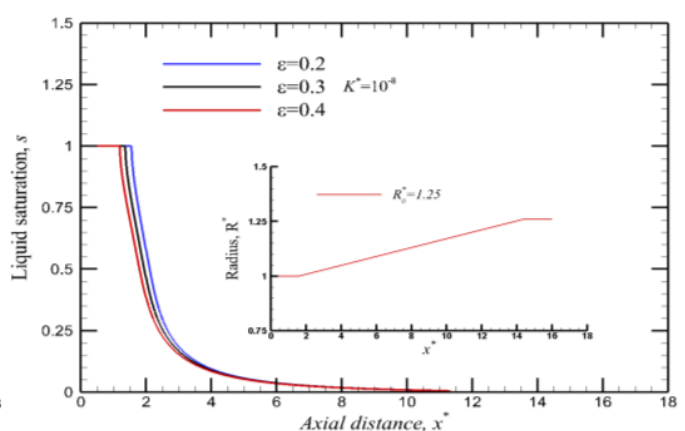

(b)

Fig. 7 Varaitions of axial a) $T$ and b) $s$ for various $\varepsilon$ at $l_{D}^{*}=0.8 L^{*}, Q_{w}^{*}=8.5 \times 10^{-1}, K^{*}=10^{-8}$ and $R e_{l}=20$

\subsection{Effects of Diffuser Geomerty}

Temperature results are presented in Fig. 8 versus various values of $l_{D}^{* *}, R_{o}^{*}$ and $L^{*}$. It is clear from the figure that $l_{D}^{*}, R_{o}^{*}$ and $L^{*}$ are important on the completion of evaporation process. With an increase in $l_{D}^{*}$ and $R_{o}^{*}$, the size of resultant region is reduced and the area of superheated is increased due to increase the heated area while keeping $Q_{w}^{*}$ fixed that will rise up heat to the medium from the evaporator wall. The results also demonstrated that the quality of superheated steam at the outlet of diffuser is considerable sensitive to diffuser geometry. Therefore, the dimensions of the diffuser shall be precisely selected to keep $T_{\max }$ below the melting temperature of metal that form the structure of porous media. 


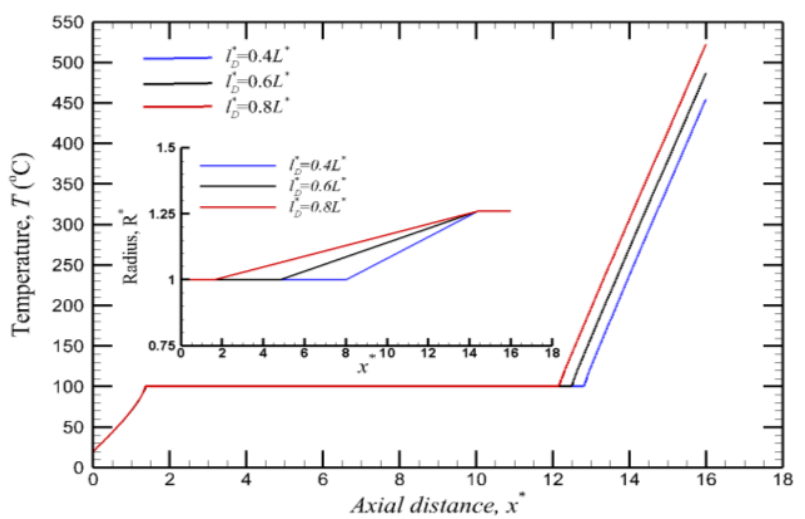

(a)

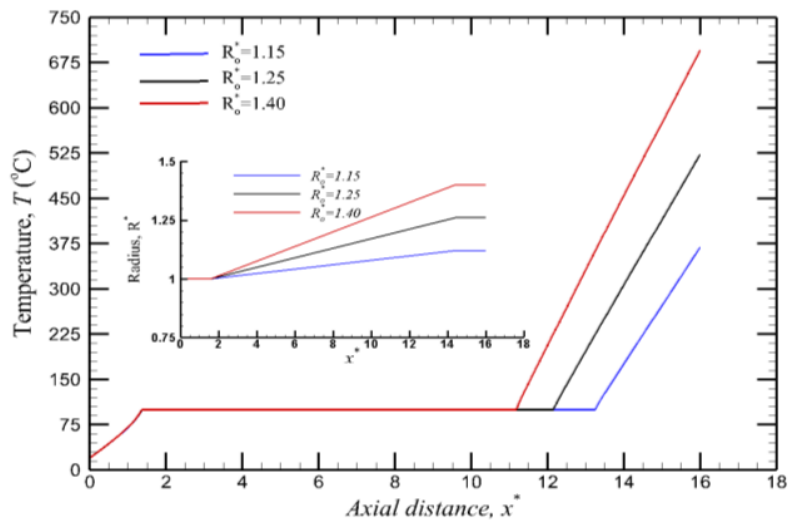

(b)

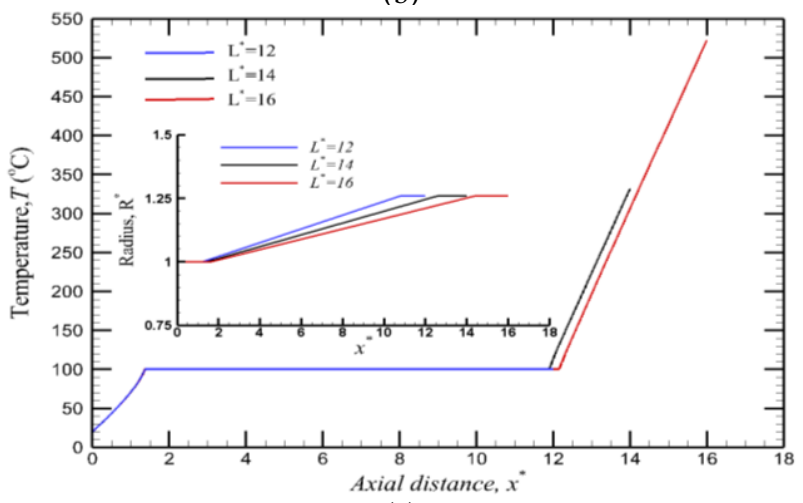

(c)

Fig. 8 Variations of axial $T$ for various a) $l_{D}^{*}$, b) $R_{o}^{*}$ and c) $L^{*}$ at $R e_{l}=20, \varepsilon=0.3, Q_{w}^{*}=8.5 \times 10^{-1}$ and $K^{*}=10^{-8}$ 


\section{Conclusions}

The following are summarized concluded remarks:

1. The results indicated that heat flux, Reynolds number, outlet radius and length of diffuser section have strong impact on the temperature profiles in the diffuser, whereas the effect porosity and Darcy number have slight influence.

2. The steam quality at the outlet of diffuser is considerable sensible to the shape of diffuser. Thus, the dimensions of the diffuser must be carefully chosen to retain maximum temperature below the melting point of the metal.

3. The whole process cycle to change the phase strongly depends on the geometric parameters of diffuser.

4. The analysis methodology applied in this work provides a helpful tool to design a diffuser filled by porous medium to keep the system under safety conditions. It should be noted that the applied test to finalize the evaporation process inside porous evaporator.

\section{References}

[1] C.Y. Wang and C. Beckermann, A two-phase mixture model of liquid-gas flow and heat transfer in capillary porous media, I. Formulation, Int. J. Heat Mass Transfer 36 (11) (1993) 2747 - 2758.

[2] C.Y. Wang, A fixed-grid numerical algorithm for two-phase flow and heat transfer in porous media, Numer. Heat Transfer, Part B. 32 (1997) 85-105.

[3] M. Najjari, S.B. Nasrallah, Heat transfer between a porous layer and a forced flow: influence of layer thickness, Drying Technol. 27 (2009) 336-343.

[4] H.Y. Li, K.C. Leong, L.W. Jin, J.C. Chai, Transient two-phase flow and heat transfer with localized heating in graphite foams, Int. J. Therm. Sci. 49 (2010) 1115-1127.

[5] H.Y. Li, K.C. Leong, L.W. Jin, J.C. Chai, Transient behavior of fluid flow and transfer with phase change in vertical porous media, Int. J. Heat Mass Transfer. 53 (2010) 5209-5222.

[6] C. Xin, Z. Rao, X. You, Z. Song, D. Han, Numerical investigation of vapor-liquid heat and mass transfer in porous media, Energy Conversion and Management. 78 (2014) 1-7.

[7] F. He, J. Wang, Numerical investigation on critical heat flux and coolant volume required for transpiration cooling with phase change, Energy Conversion and Management. 80 (2014) 591-597.

[8] O. R. Alomar, M.A.A. Mendes, D. Trimis, S. Ray, Numerical simulation of complete liquid-vapour phase change process inside porous media using smoothing of diffusion coefficient, Int. J. of Thermal Sciences. 86 (2014) 408-420.

[9] O. R. Alomar, M.A.A. Mendes, D. Trimis, S. Ray, Simulation of complete liquid-vapor phase change inside divergent porous evaporator, Int. J. of Material, Mechanics and Manufacturing. 2 (3) (2014) 223-229.

[10] O. R. Alomar, D. Trimis, S. Ray, Numerical simulation of complete liquid-vapour phase change process inside an annular porous evaporator, $10^{\text {th }}$ international conference on Freiberg-St. Petersburg Colloquium of Young Scientists, 2015. 
[11] O. R. Alomar, M.A.A. Mendes, D. Trimis, S. Ray, Simulation of complete liquid-vapour phase change process inside porous evaporator using local thermal non-equilibrium model, Int. J. of Thermal Sciences. 94 (2015) 228-241.

[12] O. R. Alomar, M. A.A. Mendes, D. Trimis, S. Ray, Numerical simulation of complete liquid-vapour phase change process inside porous media: A comparison between local thermal equilibrium and non-equilibrium models, Int. J. of Thermal Sciences. 112 (2017) $222-241$.

[13] S. Ray, O. R. Alomar, Simulation of liquid-vapour phase change process inside porous media using modified enthalpy formulation, Int. J. of Thermal Sciences. 105 (2016) 123 - 136.

[14] O. R. Alomar, M. A.A. Mendes, S. Ray, D. Trimis, Numerical investigation of complete evaporation process inside porous media using staggered and non-staggered grid arrangements, Int. J. of Thermal Sciences. 129 (2018) $56-72$.

[15] O. R. Alomar, R. R. Mohammed. M.A.A. Mendes, S. Ray, D. Trimis, Numerical investigation of two-phase flow in anisotropic porous evaporator, Int. J. of Thermal Sciences. 135 (2019) 1 - 16.

[16] O. R. Alomar, R. R. Mohammed. K. H. Mohammed, Numerical investigation of boiling and forced convection in inclined porous enclosure using modified enthalpy formulation, MATEC Web of Conferences, 240 (2018) 01001.

[17] O. R. Alomar, Modeling and Simulation of Complete Liquid-Vapor Phase Change Process inside Porous Media, Cuvillier Verlag, 2016.

[18] D.A. Nield, A. Bejan, Convection in Porous Media, third ed. Springer, New York, 2006.

[19] S.V. Patankar, Numerical Heat Transfer and Fluid Flow, Hemisphere, New York, (1980).

[20] C. Borgnakke, R. E. Sonntag, “Fundamentals of Thermodynamics,” John Wiley \& Sons, 2009 [13] P. Wildi-Tremblay, L. Gosselin, Minimizing shell-and-tube heat exchanger cost with genetic algorithms and considering maintenance, International Journal of Energy Research 31 (9) (2007) $867-885$. 